\title{
Aktuelle Empfehlungen zur Anwendung eines NIPT-Testes
}

\section{Liebe Leserinnen und Leser des Journals „Ultraschall in der Medizin“,}

seit dem 1. April 2020 ist mir die ehrenvolle Aufgabe zuteil geworden, im Team der Herausgeber unseres bewährten Journals „Ultraschall in der Medizin“ mitwirken zu dürfen. Diese persönliche Herausforderung fällt nun in eine Zeit, die durch den Ausnahmezustand der SARS-CoV-2-bedingten Pandemie geprägt wird. Vieles, was uns bis dato selbstverständlich erschien, wird nun infrage gestellt und Prioritäten haben sich in enormer Geschwindigkeit radikal verändert. Prägend für die aktuellen Wochen ist unter anderem eine in dieser Intensität nie dagewesene Verzahnung zwischen Wissenschaft, Politik und gesellschaftlichem Leben: Pressekonferenzen des RKI werden zum medialen Höhepunkt des Tages, selbst die Boulevardpresse bringt epidemiologische Grafiken auf dem Titelblatt und teils kleine virologische Studien werden zeitnah von Politikern in Fernsehansprachen zitiert. Hierin spiegelt sich meines Erachtens in besonderem Maße die Verantwortung der wissenschaftlichen Publikationsorgane wider, denn nur durch eine zeitnahe Bearbeitung, kritische Auseinandersetzung und kompetente Selektion können wissenschaftliche Neuigkeiten auch in einer politisch-gesellschaftlichen Diskussion genutzt werden. Ich hoffe hierzu einen bescheidenen Beitrag leisten zu können.
Unabhängig von dem dominierenden Pandemie-Thema möchte ich im Folgenden auf eine aktuelle Empfehlung der Sektion Gynäkologie und Geburtshilfe hinweisen. Diese bezieht sich auf die Anwendung der zellfreien DNA-Analyse, auch als nichtinvasiver Pränataltest (NIPT) bezeichnet. Die Empfehlung wurde vom Board der Sektion in Absprache mit dem DEGUM-Vorstand entworfen. In den aktuellen berufspolitischen Entscheidungen bezüglich dieses insbesondere für die Detektion der Trisomie 21 entworfenen Screeningtests wurden Stellungnahmen und Einwände seitens der Pränataldiagnostiker wenig berücksichtigt. Das Kernproblem liegt darin, dass der NIPT

\section{GOLDENE REGELN FÜR DIE DURCHFÜHRUNG EINES NIPT-TESTS}

\section{Board d. Sektion Gyn/Geb, 17.02.2020}

1. NIPT erfordert eine ärztliche Aufklärung und genetische Beratung nach dem Gendiagnostikgesetz (GenDG).

2. NIPT erlaubt derzeit zuverlässige Aussagen zur Wahrscheinlichkeit einer Trisomie 21, 18 und 13, aber keine Aussagen zu strukturellen Fehlbildungen. Diese machen jedoch den Großteil der perinatal relevanten Anomalien aus. Auch lassen sich die meisten anderen Chromosomenstörungen und syndromalen Erkrankungen nicht erkennen.

3. NIPT erfordert eine qualifizierte Ultraschalluntersuchung, idealerweise vor der Blutabnahme und nach der 12. SSW.

4. Bei sonografisch nachgewiesenen Fehlbildungen oder erhöhter Nackentransparenz ist die diagnostische Punktion (CVS oder Amniozentese) Mittel der Wahl, um Chromosomenstörungen erkennen zu können und einen unnötigen Zeitverlust bis zur endgultigen Diagnose zu vermeiden.

5. Im Rahmen einer NIPT-Untersuchung sollte grundsätzlich der fetale bzw. schwangerschaftsspezifische Anteil an der zellfreien DNA angegeben werden. Die „Fetal Fraction“ ist ein Qualitätsparameter mit großem Einfluss auf die Testgüte.

6. Ein ergebnisloser NIPT ist ein abklärungsbedürftiger Befund. In diesem Kollektiv finden sich mehr Chromosomenstörungen, insbesondere Trisomien 13 und 18 sowie Triploidien.

7. NIPT ist ein Screening-Test. Bei einem auffälligen NIPT ist eine diagnostische Punktion obligat anzubieten. Die Indikationsstellung zum Schwangerschaftsabbruch darf nicht auf einem isolierten NIPT-Befund beruhen.

8. NIPT auf Veränderungen der Geschlechtschromosomen sollten nicht routinemäßig durchgeführt werden.

9. Der Einsatz von NIPT zur Bestimmung des Risikos für seltene autosomale Aneuploidien und strukturelle Chromosomenstörungen, insbesondere Mikrodeletionen und monogenetische Erkrankungen beim Fötus, kann derzeit nicht generell empfohlen werden.

10. Bei Zwillingsschwangerschaften, nach künstlicher Befruchtung und bei Adipositas hat NIPT eine höhere Versagerquote und es liegen nur eingeschränkt Daten zur Testgüte vor. 
als Alternative und Ablösung des Ersttrimester-Screenings wie auch der diagnostischen Punktionen etabliert wird. Hierbei wird ignoriert, dass die Zielsetzung des Ersttrimester-Screenings weit über die Erkennung der Trisomie 21 hinausgeht. Schwangere sind in der Regel überrascht zu hören, dass $90 \%$ der fetalen bzw. kindlichen Fehlbildungen, wie z. B. die Spina bifi$\mathrm{da}$, in der Regel nicht chromosomalen Ursprungs sind. Auch die Verbreitung des frühen Präeklampsie-Screenings mit der Option einer ASS-basierten Prävention droht ins Abseits gedrängt zu werden. Nicht zuletzt entspricht der NIPT nicht den Kriterien eines diagnostischen Tests. Daher ist der gezielte Einsatz diagnostischer Punktionen weiterhin erforderlich.
In den letzten Jahren wurden auch in „Ultraschall in der Medizin“ hervorragende Empfehlungen und Publikationen zu diesem Thema veröffentlicht. Gegen die aktuell bedenklichen Entwicklungen in der Qualität der Ersttrimester-Diagnostik konnten sie jedoch nur begrenzt Einfluss nehmen.

Aus diesem Grund haben sich die Sektionsleitung und das Board der Sektion Gynäkologie und Geburtshilfe in Zusammenarbeit mit dem Vorstand der DEGUM dazu entschlossen, die Kernbotschaften in „10 goldene Regeln für die Durchführung eines NIPT-Tests“ zu komprimieren. Diese sollen allen in der Schwangerschaftsvorsorge mit dem Thema involvierten Kollegen als Hilfestellung dienen. Sie wurden aktuell auf der
DEGUM-Homepage veröffentlicht (https:// www.degum.de/fileadmin/dokumente/sek tionen/gynaekologie/Informationen_zum_ Fach/NIPT-10-goldene-Regeln_AK_v202002-17.pdf).

Wir möchten sie aufgrund ihrer zentralen Bedeutung auch in der „Ultraschall in der Medizin " darlegen und hoffen auf diese Art und Weise eine möglichst große Breite an NIPT-anwendenden Kollegen erreichen zu können.

Ich freue mich nun auf die weitere Zeit als Mitherausgeber der „Ultraschall in der Medizin“.

Ihr Markus Hoopmann 